\title{
Zur Ökonomisierung der Medizin (v)
}

\section{On the Economization of Medicine}

\author{
Gerd Folkers \\ Departement Geistes-, Sozial- und Staatswissenschaften, ETH Zürich
}

Die folgenden Gedanken beschäftigen sich mit einem Teilaspekt, wenn nicht einem aktuell dominierenden der Medizin, dem der medizinischen Forschung. Die Vermessung des Menschen und die Bezugnahme der Messresultate auf einen Begriff der «Gesundheit» eines Individuums ist tägliche Praxis auch bei der Hausärztin/beim Hausarzt. Nicht dass dies nicht von alters her Brauch gewesen wäre. Fiebermessung, Urinbeschau und alle Arten organoleptischer Prüfung gehörten wohl schon seit frühen Zeiten zur Feststellung von Krankheiten. Die gewaltige Änderung in Quantität und Qualität der messenden Beschreibung eines individuellen Zustands ist einer Koevolution von Humanbiologie, Messtechnik und Digitalisierung geschuldet. Eine der Schlüsselfiguren für diese Entwicklung war der vielerorts für seine Visionen gerühmte Vannevar Bush, der sich 1945 in seinem einflussreichen Essay «Science The Endless Frontier» für ein Nachkriegsprogamm der erfolgreichen «molekularen Medizin» einsetzte, das ausschliesslich auf «hard sciences» beruhte. Molekulare Medizin dieser Zeit war die von militärischer Strategie getriebenen Entwicklung von Antibiotika, Blutersatzmitteln und Insektiziden [1]. Hier entstand eine der Denkfiguren der Verknüpfung von Medizin mit der Grundlagenforschung als Voraussetzung für etwas «Nützliches». Geisteswissenschaften schloss V. Bush aus: I have a great reservation about these studies where somebody goes out and interviews a bunch of people and reads a lot of stuff and writes a book and puts it on a shelf and nobody ever reads it [2].

Die Erfolge der molekularen Medizin sind schlagend. Infektionskrankheiten und Diabetes gelten als weltweit beherrschbar. Lebensbedrohliche, seltene Erkrankungen werden mit gentherapeutischen Eingriffen immer häufiger therapierbar, Organverpflanzungen sind Standard und viele chirurgische Eingriffe dank Robotik fast ambulant. Das Resultat ist die Wahrnehmung der Medizin als «Fixit»-Sparte für den Menschen. Entsprechend sind die Erwartungshaltungen. Die Reparatur ist machbar und hat ihren Preis. Die Arzt-Patienten-Beziehung gestaltet sich zu einer Dienstleister-Kunden-Beziehung um.

Dementsprechend sind die Protagonisten der molekularen Medizin höchst angesehen. Im akademischen Spiel der Karrieren belegen diejenigen die vordersten Plätze, die Grundlagenforschung mit Klinik am erfolgreichsten zum Vorteil der Patientinnen und Patienten, im Sinne des «fix it», zu verbinden wissen. In einem Preis-LeistungsSystem scheint es nur konsequent, dass nun alle buchhalterischen Elemente zum Tragen kommen, um die Prozesse möglichst effizient zu machen. Multiplizierbarkeit und Standardisierung sind die unausweichliche Folge. Dies nicht nur für die Patientinnen und Patienten, die sich in einer Kohorte wiederfinden, sondern auch für die Ärztinnen und Ärzte, die sich statistischen Massen unterwerfen müssen. Es gilt, objektive Kriterien für die Vergabe der Studienplätze, der Assistenzen und der Professuren zu schaffen, und endet in einer «Ranking»-Manie auf der Basis der Messbarkeit. Das nach dieser Massgabe beste Produkt, ob Antibiotikum oder Mensch, wird auf den Markt gebracht. Die Auswirkungen dieses Konzepts spüren wir heute als unbehagliche Situation der «Ökonomisierung», wie der Schweizerische Wissenschaftsrat in seiner Untersuchung «Growth of Science» mit einem Fokus auf die medizinische Forschung festhält:

... wurden in den vergangenen 20 Jahren in den führenden Wissenschaftsnationen verschiedene Massnahmen ergriffen, um eine möglichst transparente und effiziente Verwendung der öffentlichen Mittel zu gewährleisten und sicherzustellen, dass sich die Forschung an den wichtigsten sozioökonomischen Zielen orientiert. Diese beiden Entwicklungen - die Zunahme der Forschungsaktivitäten und die Einführung neuer Methoden zur Steuerung der Wissenschaft - bedeuten einen grundlegenden Wandel sowohl für die Forschenden als auch für die Gesellschaft. Sie haben insbesondere den Wettbewerb um Finanzmittel und eine beschränkte Anzahl akademischer Stellen verstärkt. Gleichzeitig bewirkten die Verfahren zur quantitativen Messung wissenschaftlicher Leistung tiefgreifende Veränderungen bei der Publikation wissenschaftlicher Arbeiten [3].

Altimetrics verzeichnet unter den 100 meist zitierten wissenschaftlichen Veröffentlichungen im Jahr 2018 allein 62 Artikel aus der medizinischen Forschung [4]. Wer es in diese Liste schafft, geniesst hohes Ansehen und damit einen hohen Marktwert. Denkansätze, die sich nicht diesen Kriterien unterordnen, haben es schwer.

Das deutet aber nicht auf einen negativen Effekt des Wettbewerbs, der Ökonomie, sondern eher auf ein Phänomen der Wettbewerbsverzerrung hin. Die Neubewertung, das Scheitern, aus dem man lernt, scheint speziell in der medizinischen Forschung ein langer Prozess zu sein. Das «fail and fail fast» der reinen technikgetriebenen Wissenschaften verbietet sich schon aus ethischen Gründen. Umso wichtiger ist es, die Bewertungskriterien zu refor- 
mieren und der Medizin anzupassen, anstatt pauschal eine «Ökonomisierungsideologie» zu etablieren. Hier ist ein Umdenken in der staatlichen Förderung ebenso gefragt wie in den privaten «science industries». Im Idealfall sind akademische Institutionen komplementär zur industriellen Forschung, denn sie geniessen - theoretisch - das Privileg des hohen Risikos, wenn es denn auch Rahmenbedingungen gäbe, die ein Scheitern auf hohem Niveau förderten. Dieses Scheitern auf hohem Niveau müsste aber auch genauso prominent publizierbar sein, wie die Erfolgsgeschichten, sonst lernen wir nichts. Historische, soziologische, psychologische, philosophische und, natürlich, ökonomische Perspektiven gehören auf gleichem Niveau zur medizinischen Forschung. Leider ereilt diese Zweige oft ein Feigenblattdasein, denn nicht überall ist Interdisziplinarität drin, wo sie plakativ gefördert wird. Die Verankerung gesicherten medizinischen Wissens ist eine hochkomplexe Aufgabe [5]. Richtlinien, systematische Übersichtsartikel, neue und «veraltete» Lehrbücher in die Kontexte einzuordnen und zu bewerten erfordert Teilnahme aller Wissenschaften.

Solange deshalb das Einwerben bestimmter Drittmittel und die Publikation in bestimmten Zeitschriften zur ausschliesslichen Einstellungsvoraussetzung gehört und überall standardisierte Evaluationsmechanismen greifen, wird die Wettbewerbsverszerrung Bestand haben und eine positive Ökonomisierung verhindern.

\section{Bibliografie}

1. de Chadarevian S, Kamming S (Eds.): Molecularizing biology and medicine: New practices and alliances 1910s-1970s. Reading; Harwood Academic Publishers: 1998.

2. Zachary GP: Endless Frontier: Vannevar Bush, Engineer of the American Century. New York; Free Press: 1997. S. 96.

3. Schweizer Wissenschaftsrat SWR: The growth of science: Implications for the evaluation and funding of research in Switzerland. Policy analysis and recommendations by the Swiss Science Council SSC. Empirical analysis by B. Hendriks, M. Reinhart, and C. Schendzielorz, German Centre for Higher Education Research and Science Studies (DZHW), Berlin. Bern; Swiss Science Council: 2018. https://www.swir.ch/de/publika tionen-de; letzter Zugriff: 10.12.2019.

4. Altimetric. London: 2018. https://www.altmetric.com/top100/ 2018/; letzter Zugriff: 10.12.2019.

5. Catillon M: Medical knowledge synthesis: A brief overview. Cambridge, MA; The National Bureau of Economic Research: 2017. http://www.nber.org/aging/valmed/WhitePaper-Catillon 10.2017.pdf; letzter Zugriff: 10.12.2019.

\section{Prof. Dr. Gerd Folkers}

ETH Zürich

Departement Geistes-, Sozial- und Staatswissenschaften

Rämistrasse 101

8092 Zürich

gerd.folkers@gess.ethz.ch 